“C 2018 IEEE. Personal use of this material is permitted. Permission from IEEE must be obtained for all other uses, in any current or future media, including reprinting/republishing this material for advertising or promotional purposes, creating new collective works, for resale or redistribution to servers or lists, or reuse of any copyrighted component of this work in other works." 


\title{
Quantum Channel Simulation and the Channel's Smooth Max-Information
}

\author{
Kun Fang ${ }^{1},{ }^{*}$ Xin Wang $^{1}$, Marco Tomamichel ${ }^{1}$, and Mario Berta ${ }^{2}$ \\ ${ }^{1}$ Centre for Quantum Software and Information, \\ Faculty of Engineering and Information Technology, \\ University of Technology Sydney, NSW 2007, Australia and \\ 2 Department of Computing, Imperial College London, London, United Kingdom
}

\begin{abstract}
We study the general framework of quantum channel simulation, that is, the ability of a quantum channel to simulate another one using different classes of codes. First, we show that the minimum error of simulation and the one-shot quantum simulation cost under no-signalling assisted codes are given by semidefinite programs. Second, we introduce the channel's smooth max-information, which can be seen as a one-shot generalization of the mutual information of a quantum channel. We provide an exact operational interpretation of the channel's smooth max-information as the oneshot quantum simulation cost under no-signalling assisted codes. Third, we derive the asymptotic equipartition property of the channel's smooth max-information, i.e., it converges to the quantum mutual information of the channel in the independent and identically distributed asymptotic limit. This implies the quantum reverse Shannon theorem in the presence of no-signalling correlations. Finally, we explore the simulation cost of various quantum channels.
\end{abstract}

\section{INTRODUCTION}

Channel simulation is a fundamental problem in information theory. It asks how to use a (noisy) channel from Alice (A) to Bob (B) to simulate another (noisy) channel also from A to B [1]. Depending on the different resources available between $A$ and $B$, this simulation problem has many variants.

For classical channels, Shannon's noisy channel coding theorem determines the capability of noisy classical channels to simulate noiseless ones [2]. Dual to this famous coding theorem, the 'reverse Shannon theorem' concerns the use of noiseless channels to simulate noisy ones [3]. Specifically, every channel can be simulated using an amount of classical communication equal to the capacity of the channel when there is free shared randomness between A and B in the asymptotic setting [3]. For quantum channels, the case when A and B share an unlimited amount of entanglement has been completely solved by the quantum reverse Shannon theorem (QRST) [4, 5], which states that the rate to optimally simulate a quantum channel in the asymptotic setting is determined by its entanglement-assisted classical capacity. In the zero-error scenario [6], using one channel to simulate another exactly with the aid of no-signalling correlations has been studied recently in [7-9]. The problem of quantum channel simulations via other quantum resources has also been investigated in $[10,11]$.

In realistic settings, the number of channel uses is necessarily limited, and it is not easy to perform encoding and decoding circuits coherently over a large number of qubits in the near future. Therefore, it is important to characterize how well we can simulate a quantum channel from another with finite resources. The first step in this direction is to consider the one-shot setting. One-shot analysis has recently attracted great interest in classical information theory (see, e.g., $[12,13]$ ) and quantum information theory (see, e.g., [14-22]). In one-shot information theory, the smooth max-information of a quantum state [5] and its generalizations [23] are all basic and useful quantities, which have various applications in quantum rate distortion theory as well as the physics of quantum many-body systems.

In this work, we focus on quantum channel simulation in both the one-shot and the asymptotic regime. The central quantity we introduce is the channel's smooth max-information. Our results can be summarized

*Electronic address: kun.fang-1@ @ student.uts.edu.au 
as follows. In Section III, we develop a framework for quantum channel simulation assisted with different codes in the one-shot regime, where one has access only to a single use of the quantum channel. In particular, we characterize the minimum error of channel simulation under no-signalling (NS) and positivepartial-transpose-preserving (PPT) codes as semidefinite programs (SDPs) [24]. The cost of approximately simulating a channel via noiseless quantum channels under NS-assisted codes can also be characterized as an SDP. In Section IV, we introduce the channel's smooth max-information, which can be seen as a oneshot generalization of the mutual information of a quantum channel. Notably, this newly introduced entropy has the exact operational interpretation as the one-shot quantum simulation cost under NS-assisted codes. Then we prove its asymptotic equipartition property which directly implies the quantum reverse Shannon theorem in the presence of no-signalling correlations.

In the setting of the entanglement-assisted one-shot capacity of quantum channels, Matthews and Wehner gave a converse bound in terms of the channel's hypothesis testing relative entropy [17]. Moreover, a subset of us recently showed that the activated NS-assisted one-shot capacity is exactly given by the channel's hypothesis testing relative entropy [25] - generalizing the corresponding classical results [12,26]. This suggests that the operational min- and max-type one-shot analogues of the channel's mutual information are the channel's hypothesis testing relative entropy and the channel's smooth max-information, respectively.

In Section V, as applications, we evaluate the cost of simulating fundamental quantum channels with finite resources. In particular, we derive a linear program to evaluate the finite blocklength simulation cost of quantum depolarizing channels.

\section{CHANNEL SIMULATION AND CODES}

Let us now formally introduce the task of channel simulation and some basic notations. A quantum channel (quantum operation) $\mathcal{N}_{A_{o} \rightarrow B_{i}}$ is a completely positive (CP) and trace-preserving (TP) linear map from operators on a finite-dimensional Hilbert space $A_{o}$ to operators on a finite-dimensional Hilbert space $B_{i}$. As shown in Fig. 1, Alice and Bob share a quantum channel $\mathcal{N}_{A_{o} \rightarrow B_{i}}$. By adding encoding and decoding scheme, they can use the channel $\mathcal{N}$ to simulate another channel $\mathcal{M}$. Composing with the encoder and decoder, their effective channel is given by $\widetilde{\mathcal{N}}_{A_{i} \rightarrow B_{o}}=\Pi_{A_{i} B_{i} \rightarrow A_{o} B_{o}} \circ \mathcal{N}_{A_{o} \rightarrow B_{i}}$, where $\Pi$ is a bipartite quantum operation that generalizes the usual encoding scheme $\mathcal{E}$ and decoding scheme $\mathcal{D}$. (Note that the bipartite quantum operation $\Pi$ here is required to be $\mathrm{B}$ to A no-signalling, which makes the composition of $\Pi$ and $\mathcal{N}$ feasible $[8,27]$.) We say such $\Pi$ is an $\Omega$-assisted code if it can be implemented by local operations with $\Omega$-assistance. In the following, we eliminate $\Omega$ for the case of unassisted codes. We write $\Omega=\mathrm{NS}$ and $\Omega=$ PPT for NS-assisted and PPT-assisted codes, respectively [28-32].

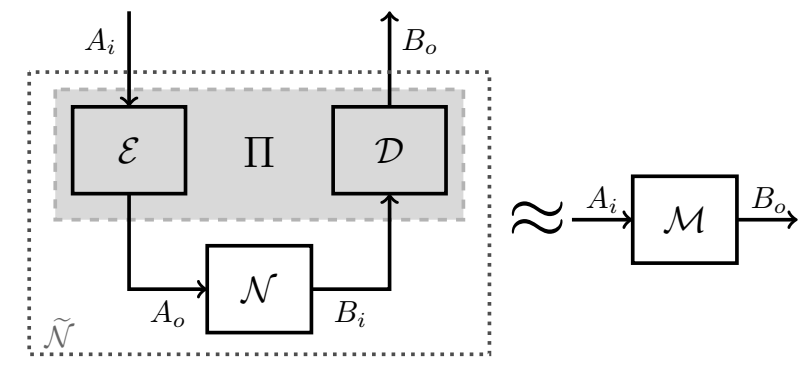

FIG. 1: General framework of quantum channel simulation.

In particular,

- an unassisted code reduces to the product of encoder and decoder, i.e., $\Pi=\mathcal{D}_{B_{i} \rightarrow B_{o}} \mathcal{E}_{A_{i} \rightarrow A_{o}}$; 
- a NS-assisted code corresponds to a bipartite quantum operation which is no-signalling from Alice to Bob and vice-versa;

- a PPT-assisted code corresponds to a bipartite operation whose Choi-Jamiołkowski matrix is positive under partial transpose over systems $B_{i} B_{o}$.

For any two quantum channels $\mathcal{N}$ and $\mathcal{M}$, the minimum error of simulation from $\mathcal{N}$ to $\mathcal{M}$ under $\Omega$ assisted codes is defined as

$$
\omega_{\Omega}(\mathcal{N}, \mathcal{M}):=\frac{1}{2} \inf _{\Pi \in \Omega}\|\Pi \circ \mathcal{N}-\mathcal{M}\|_{\diamond}
$$

where $\|\mathcal{F}\|_{\diamond}:=\sup _{k \in \mathbb{N}}\left\|\mathcal{F} \otimes \operatorname{id}_{k}\right\|_{1}$ denotes the diamond norm and $\|\cdot\|_{1}$ is induced by the Schatten 1-norm. The channel simulation rate from $\mathcal{N}$ to $\mathcal{M}$ under $\Omega$-assisted codes is defined as

$$
S_{\Omega}(\mathcal{N}, \mathcal{M}):=\lim _{\varepsilon \rightarrow 0} \inf \left\{\frac{n}{m} \mid \omega_{\Omega}\left(\mathcal{N}^{\otimes n}, \mathcal{M}^{\otimes m}\right) \leq \varepsilon\right\},
$$

where the infimum is taken over ratios $\frac{n}{m}$ with $n, m \in \mathbb{N}$. In this framework of channel simulation, the classical capacity $C(\mathcal{N})$ and the quantum capacity $Q(\mathcal{N})$ of the channel $\mathcal{N}$ are given by

$$
C(\mathcal{N})=S\left(\mathcal{N}, \widehat{\mathrm{id}}_{2}\right)^{-1} \text { and } Q(\mathcal{N})=S\left(\mathcal{N}, \mathrm{id}_{2}\right)^{-1}
$$

where $\widehat{\mathrm{id}}_{2}$ is the one-bit noiseless channel and $\mathrm{id}_{2}$ is the one-qubit noiseless channel.

If we consider simulating the given channel $\mathcal{N}$ via a $m$-dimensional noiseless quantum channel $\mathrm{id}_{m}$, then the one-shot $\varepsilon$-error quantum simulation cost under $\Omega$-assisted codes is defined as

$$
S_{\Omega, \varepsilon}^{(1)}(\mathcal{N}):=\log \inf \left\{m \in \mathbb{N} \mid \omega_{\Omega}\left(\operatorname{id}_{m}, \mathcal{N}\right) \leq \varepsilon\right\},
$$

where the logarithms in this work are taken in the base two. The asymptotic quantum simulation cost is given by the regularization

$$
S_{\Omega}(\mathcal{N})=\lim _{\varepsilon \rightarrow 0} \lim _{n \rightarrow \infty} \frac{1}{n} S_{\Omega, \varepsilon}^{(1)}\left(\mathcal{N}^{\otimes n}\right)
$$

\section{CHANNEL SIMULATION VIA NOISY QUANTUM CHANNELS}

Based on the definitions in the above section, we show that the minimum error of simulation under NS-assisted (and PPT-assisted) codes can be given by SDPs. The one-shot $\varepsilon$-error quantum simulation cost under NS-assisted codes can also be given by an SDP. These SDPs can be easily implemented for small blocklength and they also lay the foundation of analysis in the following sections.

Proposition 1 For any two quantum channels $\mathcal{N}$ and $\mathcal{M}$ with corresponding Choi-Jamiotkowski matrices $J_{\mathcal{N}}$ and $J_{\mathcal{M}}$, the minimum error of simulation from $\mathcal{N}$ to $\mathcal{M}$ under $N S$-assisted codes $\omega_{\mathrm{NS}}(\mathcal{N}, \mathcal{M})$ is given by the following $S D P$,

$$
\begin{array}{lll}
\text { inf } & \gamma \\
\text { s.t. } & \operatorname{Tr}_{B_{o}} Y_{A_{i} B_{o}} \leq \gamma \mathbb{1}_{A_{i}}, & \\
& Y_{A_{i} B_{o}} \geq J_{\widetilde{\mathcal{N}}}-J_{\mathcal{M}}, Y_{A_{i} B_{o}} \geq 0, & \\
& J_{\widetilde{\mathcal{N}}}=\operatorname{Tr}_{A_{o} B_{i}}\left(J_{\mathcal{N}}^{T} \otimes \mathbb{1}_{A_{i} B_{o}}\right) J_{\Pi}, & \\
& J_{\Pi} \geq 0, \operatorname{Tr}_{A_{o} B_{o}} J_{\Pi}=\mathbb{1}_{A_{i} B_{i}}, & (\mathrm{CP}, \mathrm{TP}) \\
& \operatorname{Tr}_{A_{o}} J_{\Pi}=\mathbb{1}_{A_{i}} /\left|A_{i}\right| \otimes \operatorname{Tr}_{A_{o} A_{i}} J_{\Pi}, & (\mathrm{A} \not \mathrm{B}) \\
& \operatorname{Tr}_{B_{o}} J_{\Pi}=\mathbb{1}_{B_{i}} /\left|B_{i}\right| \otimes \operatorname{Tr}_{B_{i} B_{o}} J_{\Pi} . & (\mathrm{B} \not \rightarrow \mathrm{A})
\end{array}
$$


To obtain $\omega_{\mathrm{NS} \cap \mathrm{PPT}}(\mathcal{N}, \mathcal{M})$, we only need to add the PPT constraint $J_{\Pi}^{T_{B_{i} B_{o}}} \geq 0$, where $T_{B_{i} B_{o}}$ denotes the partial transpose over systems $B_{i} B_{o}$.

Proof Note that for any two quantum channels $\mathcal{N}_{1}, \mathcal{N}_{2}$ from $A$ to $B$, the diamond norm of their difference can be expressed as an SDP of the following form [33]:

$$
\frac{1}{2}\left\|\mathcal{N}_{1}-\mathcal{N}_{2}\right\|_{\diamond}=\inf \left\{\gamma \mid \operatorname{Tr}_{B} Y \leq \gamma \mathbb{1}_{A}, Y \geq J_{\mathcal{N}_{1}}-J_{\mathcal{N}_{2}}, Y \geq 0\right\},
$$

where $J_{\mathcal{N}_{1}}$ and $J_{\mathcal{N}_{2}}$ are the corresponding Choi-Jamiołkowski matrices. We denote the Choi-Jamiołkowski matrix of code $\Pi$ as $J_{\Pi}$. Following similar steps in $[8,28]$, the Choi-Jamiołkowski matrix of the effective channel $\widetilde{\mathcal{N}}=\Pi \circ \mathcal{N}$ is given by

$$
J_{\widetilde{\mathcal{N}}}=\operatorname{Tr}_{A_{o} B_{i}}\left(J_{\mathcal{N}}^{T} \otimes \mathbb{1}_{A_{i} B_{o}}\right) J_{\Pi}
$$

Together with the constraints of the code $\Pi$, we have the resulting SDP (6). The constraints in Eq. (6e) represent the CP and TP conditions of the bipartite operation $\Pi$. The constraints in Eqs. (6f) and (6g) represent the no-signalling conditions that A cannot signal to B and B cannot signal to A, respectively.

Corollary 2 The minimum error to simulate a quantum channel $\mathcal{N}$ via a noiseless quantum channel under $N S$-assisted codes $\omega_{\mathrm{NS}}\left(\mathrm{id}_{m}, \mathcal{N}\right)$ is given by the following SDP,

$$
\begin{array}{ll}
\text { inf } & \gamma \\
\text { s.t. } & \operatorname{Tr}_{B} Y_{A B} \leq \gamma \mathbb{1}_{A}, \\
& Y_{A B} \geq J_{\widetilde{\mathcal{N}}}-J_{\mathcal{N}}, Y_{A B} \geq 0 \\
& J_{\widetilde{\mathcal{N}}} \geq 0, \operatorname{Tr}_{B} J_{\widetilde{\mathcal{N}}}=\mathbb{1}_{A}, \\
& J_{\widetilde{\mathcal{N}}} \leq \mathbb{1}_{A} \otimes V_{B}, \operatorname{Tr} V_{B}=m^{2} .
\end{array}
$$

To obtain $\omega_{\mathrm{NS \cap PPT}}\left(\mathrm{id}_{m}, \mathcal{N}\right)$, we only need to add the PPT constraint $-\mathbb{1}_{A} \otimes V_{B}^{T} \leq m J_{\widetilde{\mathcal{N}}}^{T_{B}} \leq \mathbb{1}_{A} \otimes V_{B}^{T}$.

Proof The main idea to do the simplification is to utilize the symmetry of the noiseless quantum channel. Denote the Choi-Jamiołkowski matrix of $\mathrm{id}_{m}$ as $J_{m}=\sum_{i, j=0}^{m-1}|i i\rangle\left\langle\left. j j\right|_{A_{o} B_{i}}\right.$. Then $J_{m}$ is invariant under any local unitary $U_{A_{o}} \otimes \bar{U}_{B_{i}}$.

Suppose $J_{\Pi}$ is optimal for SDP (6), we can check that $\left(U_{A_{o}} \otimes \bar{U}_{B_{i}}\right) J_{\Pi}\left(U_{A_{o}} \otimes \bar{U}_{B_{i}}\right)^{\dagger}$ is also optimal. Any convex combination of optimal solutions remains optimal. Thus, without loss of generality we can take

$$
J_{\Pi}=\int d U\left(U_{A_{o}} \otimes \bar{U}_{B_{i}}\right) J_{\Pi}\left(U_{A_{o}} \otimes \bar{U}_{B_{i}}\right)^{\dagger}=\frac{J_{m}}{m} \otimes H_{A_{i} B_{o}}+\left(\mathbb{1}_{A_{o} B_{i}}-\frac{J_{m}}{m}\right) \otimes K_{A_{i} B_{o}},
$$

where the integral is taken over the Haar measure and $H, K$ are operators on system $A_{i} B_{o}$. Taking such form of $J_{\Pi}$ into $\operatorname{SDP}(6)$, we know that

$$
\begin{aligned}
& \text { Eq. (6d) } \Longleftrightarrow J_{\widetilde{\mathcal{N}}}=m H, \\
& \text { Eq. (6e) } \Longleftrightarrow H \geq 0, K \geq 0, \operatorname{Tr}_{B_{o}}\left(H+\left(m^{2}-1\right) K\right)=m \mathbb{1}_{A_{i}} .
\end{aligned}
$$

Since $J_{\widetilde{\mathcal{N}}}$ is the Choi-Jamiołkowski matrix of the effective channel, we have $\operatorname{Tr}_{B_{o}} J_{\widetilde{\mathcal{N}}}=\operatorname{Tr}_{B_{o}} m H=\mathbb{1}_{A_{i}}$ and thus $\operatorname{Tr}_{B_{o}} m K=\mathbb{1}_{A_{i}}$. This implies that Eq. (6g) is trivial and

$$
\text { Eq. (6f) } \Longleftrightarrow H+\left(m^{2}-1\right) K=\mathbb{1}_{A_{i}} /\left|A_{i}\right| \otimes \operatorname{Tr}_{A_{i}}\left(H+\left(m^{2}-1\right) K\right) \text {. }
$$

Denote $V_{B_{o}}=m \operatorname{Tr}_{A_{i}}\left(H+\left(m^{2}-1\right) K\right) /\left|A_{i}\right|$. We have $J_{\widetilde{\mathcal{N}}}+\left(m^{2}-1\right) m K=\mathbb{1}_{A_{i}} \otimes V_{B_{o}}$. Finally, we will obtain SDP (9) by eliminating the variable $K$ and replacing the subscript $A_{i}$ to $A, B_{o}$ to $B$. 
From the definition of one-shot quantum simulation cost and Corollary 2, we have the following result.

Proposition 3 For any quantum channel $\mathcal{N}$ and error tolerance $\varepsilon \geq 0$, the one-shot $\varepsilon$-error quantum simulation cost under NS-assisted codes is given by the following SDP,

$$
\begin{aligned}
S_{\mathrm{NS}, \varepsilon}^{(1)}(\mathcal{N})=\frac{1}{2} \log \text { inf } & \operatorname{Tr} V_{B} \\
\text { s.t. } & \operatorname{Tr}_{B} Y_{A B} \leq \varepsilon \mathbb{1}_{A}, \\
& Y_{A B} \geq J_{\widetilde{\mathcal{N}}}-J_{\mathcal{N}}, Y_{A B} \geq 0, \\
& J_{\widetilde{\mathcal{N}}} \geq 0, \operatorname{Tr}_{B} J_{\widetilde{\mathcal{N}}}=\mathbb{1}_{A}, \\
& J_{\widetilde{\mathcal{N}}} \leq \mathbb{1}_{A} \otimes V_{B} .
\end{aligned}
$$

To match the exact definition of the one-shot quantum simulation cost, we need to apply a ceiling function to the optimal value $\sqrt{\operatorname{Tr} V_{B}}$ based on SDP (14). For simplicity, we ignore this extra step in the following discussion. Note that the one-shot quantum simulation cost under NS $\cap$ PPT-assisted codes is not an SDP, since the objective function $m$ appears in the conditions $\operatorname{Tr} V_{B}=m^{2}$ and $-\mathbb{1}_{A} \otimes V_{B}^{T} \leq$ $m J_{\widetilde{\mathcal{N}}}^{T_{B}} \leq \mathbb{1}_{A} \otimes V_{B}^{T}$ with different powers. We do not see a way to obtain a linear objective function.

It is also worth mentioning that the zero-error quantum simulation cost was studied by Duan and Winter in [8]. The authors show that the zero-error NS-assisted simulation cost is given by the conditional minentropy of the channel's Choi-Jamiołkowski matrix [8, Theorem 2]. The result we obtained in Proposition 3 is more general and can reduce to the zero-error case by letting $\varepsilon=0$. More specifically, taking $\varepsilon=0$ will lead to $Y_{A B}=0$ and thus $J_{\widetilde{\mathcal{N}}}=J_{\mathcal{N}}$. Then we have

$$
\begin{aligned}
S_{\mathrm{NS}, 0}^{(1)}(\mathcal{N})=\frac{1}{2} \log \inf & \operatorname{Tr} V_{B} \\
\text { s.t. } & J_{\mathcal{N}} \leq \mathbb{1}_{A} \otimes V_{B} .
\end{aligned}
$$

Since the conditional min-entropy is additive (see [14]), we have

$$
S_{\mathrm{NS}, 0}(\mathcal{N}):=\lim _{n \rightarrow \infty} \frac{1}{n} S_{\mathrm{NS}, 0}^{(1)}\left(\mathcal{N}^{\otimes n}\right)=S_{\mathrm{NS}, 0}^{(1)}(\mathcal{N}) .
$$

\section{THE CHANNEL'S MAX-INFORMATION AND ITS ASYMPTOTIC EQUIPARTITION PROPERTY}

In this section, we introduce a novel entropy called the channel's smooth max-information and show that it has an operational interpretation regarding the quantum simulation cost of a channel. Furthermore, we prove the asymptotic equipartition property (AEP) of the channel's smooth max-information and explore its close relation to the well-known quantum reverse Shannon theorem (QRST).

Some basic notations will be used in this section. The set of sub-normalized quantum states is denoted as $S_{\leq}(A):=\{\rho \geq 0 \mid \operatorname{Tr} \rho \leq 1\}$. The set of quantum states is denoted as $S_{=}(A):=\{\rho \geq 0 \mid \operatorname{Tr} \rho=1\}$. We denote $\rho_{A}$ as the reduced state of $\rho_{A B}$, i.e. $\rho_{A}:=\operatorname{Tr}_{B} \rho_{A B}$. The purified distance [14] based on the generalized fidelity is given by

$$
P(\rho, \sigma):=\sqrt{1-F^{2}(\rho, \sigma)} \text { with } F(\rho, \sigma):=\left\|\rho^{1 / 2} \sigma^{1 / 2}\right\|_{1}+\sqrt{(1-\operatorname{Tr} \rho)(1-\operatorname{Tr} \sigma)} .
$$

We say $\rho$ and $\sigma$ are $\varepsilon$-close and write $\rho \approx^{\varepsilon} \sigma$ if and only if $P(\rho, \sigma) \leq \varepsilon$. 


\section{A. The channel's max-information}

The max-relative entropy of $\rho \in \mathcal{S}_{\leq}(A)$ with respect to $\sigma \geq 0$ is defined as $[34,35]$

$$
D_{\max }(\rho \| \sigma):=\inf \left\{t \mid \rho \leq 2^{t} \cdot \sigma\right\} .
$$

The max-information that $B$ has about $A$ for $\rho_{A B} \in \mathcal{S}_{\leq}(A B)$ is defined as [5]

$$
I_{\max }(A: B)_{\rho}:=\inf _{\sigma_{B} \in \mathcal{S}_{=(B)}} D_{\max }\left(\rho_{A B} \| \rho_{A} \otimes \sigma_{B}\right),
$$

which is a one-shot generalization of the quantum mutual information

$$
I(A: B)_{\rho}:=\inf _{\sigma_{B} \in \mathcal{S}_{=}(B)} D\left(\rho_{A B} \| \rho_{A} \otimes \sigma_{B}\right) .
$$

Definition 4 For any quantum channel $\mathcal{N}_{A^{\prime} \rightarrow B}$ we define the channel's max-information of $\mathcal{N}$ as

$$
I_{\max }(A: B)_{\mathcal{N}}:=I_{\max }(A: B)_{\mathcal{N}_{A^{\prime} \rightarrow B}\left(\Phi_{A A^{\prime}}\right)},
$$

where $\Phi_{A A^{\prime}}$ is the maximally entangled state on $A A^{\prime}$.

Remark The following argument shows that this definition does not depend on the input state $\Phi_{A A^{\prime}}$. That is, for any full rank state $\phi_{A^{\prime}}$ with a purification $\phi_{A A^{\prime}}$, we have

$$
I_{\max }(A: B)_{\mathcal{N}}=I_{\max }(A: B)_{\mathcal{N}_{A^{\prime} \rightarrow B}\left(\phi_{A A^{\prime}}\right)} .
$$

From the definitions (18), (19) and (21), we have

$$
I_{\max }(A: B)_{\mathcal{N}}=\inf \left\{t \mid \mathcal{N}_{A^{\prime} \rightarrow B}\left(\Phi_{A A^{\prime}}\right) \leq 2^{t} \cdot \frac{\mathbb{1}_{A}}{|A|} \otimes \sigma_{B}, \sigma_{B} \in \mathcal{S}_{=}(B)\right\}
$$

Since

$$
\mathcal{N}_{A^{\prime} \rightarrow B}\left(\phi_{A A^{\prime}}\right)=|A| \cdot \mathcal{N}_{A^{\prime} \rightarrow B}\left(\sqrt{\phi_{A}} \Phi_{A A^{\prime}} \sqrt{\phi_{A}}\right)=|A| \cdot \sqrt{\phi_{A}} \mathcal{N}_{A^{\prime} \rightarrow B}\left(\Phi_{A A^{\prime}}\right) \sqrt{\phi_{A}}
$$

the first condition in (23) is equivalent to $\mathcal{N}_{A^{\prime} \rightarrow B}\left(\phi_{A A^{\prime}}\right) \leq 2^{t} \cdot \phi_{A} \otimes \sigma_{B}$, which implies Eq. (22).

Comparing Eqs. (15) and (23), we can write the one-shot zero-error quantum simulation cost as the channel's max-information:

$$
S_{\mathrm{NS}, 0}^{(1)}(\mathcal{N})=\frac{1}{2} I_{\max }(A: B)_{\mathcal{N}}
$$

In the following, we show this relation beyond the zero-error case. For this, we define the smoothed version of the channel's max-information.

Definition 5 For any quantum channel $\mathcal{N}$, we define the channel's smooth max-information as

$$
\begin{aligned}
I_{\max }^{\varepsilon}(A: B)_{\mathcal{N}}:=\inf & I_{\max }(A: B)_{\widetilde{\mathcal{N}}} \\
\text { s.t. } \quad & \frac{1}{2}\|\widetilde{\mathcal{N}}-\mathcal{N}\|_{\diamond} \leq \varepsilon, \widetilde{\mathcal{N}} \in \operatorname{CPTP}\left(A^{\prime}: B\right)
\end{aligned}
$$

where $\mathrm{CPTP}\left(A^{\prime}: B\right)$ denotes the set of all CPTP maps from $A^{\prime}$ to $B$.

We show that the one-shot $\varepsilon$-error quantum simulation cost is exactly given by the channel's smooth maxinformation. This provides the operational meaning of this new entropy. 
Theorem 6 For any quantum channel $\mathcal{N}$ and given error tolerance $\varepsilon \geq 0$, it holds that

$$
S_{\mathrm{NS}, \varepsilon}^{(1)}(\mathcal{N})=\frac{1}{2} I_{\max }^{\varepsilon}(A: B)_{\mathcal{N}}
$$

Proof We first notice that the constraints in Eq. (14d) $J_{\widetilde{\mathcal{N}}} \geq 0, \operatorname{Tr}_{B} J_{\widetilde{\mathcal{N}}}=\mathbb{1}_{A}$ uniquely define a CPTP map $\widetilde{\mathcal{N}}$ due to the Choi-Jamiołkowski isomorphism. Applying the SDP of diamond norm in (7), we find

$$
\begin{aligned}
S_{\mathrm{NS}, \varepsilon}^{(1)}(\mathcal{N})=\frac{1}{2} \log \inf & \operatorname{Tr} V_{B} \\
\text { s.t. } & \frac{1}{2}\|\widetilde{\mathcal{N}}-\mathcal{N}\|_{\diamond} \leq \varepsilon, \\
& \widetilde{\mathcal{N}} \in \operatorname{CPTP}\left(A^{\prime}: B\right), \\
& J_{\widetilde{\mathcal{N}}} \leq \mathbb{1}_{A} \otimes V_{B} .
\end{aligned}
$$

From Eqs. (15) and (25), we know that

$$
I_{\max }(A: B)_{\mathcal{N}}=\log \inf \left\{\operatorname{Tr} V_{B} \mid J_{\mathcal{N}} \leq \mathbb{1}_{A} \otimes V_{B}\right\} .
$$

Combining Eqs. (28) and (29), we obtain the desired result.

Remark From this result, we can operationally verify that the data-processing inequality holds for the channel's smooth max-information, i.e.,

$$
I_{\max }^{\varepsilon}\left(A_{0}: B_{0}\right)_{\mathcal{T} \circ \mathcal{N} \circ \mathcal{F}} \leq I_{\max }^{\varepsilon}\left(A_{1}: B_{1}\right)_{\mathcal{N}}
$$

holds for any CPTP maps $\mathcal{N}_{A_{1}^{\prime} \rightarrow B_{1}}, \mathcal{F}_{A_{0}^{\prime} \rightarrow A_{1}^{\prime}}$ and $\mathcal{T}_{B_{1} \rightarrow B_{0}}$. This follows from the fact that we need less resources to simulate a quantum channel with higher noise. Furthermore, if $\mathcal{F}$ and $\mathcal{T}$ are both isometries equality holds.

\section{B. Asymptotic equipartition property and the quantum reverse Shannon theorem}

In the framework of quantum channel simulation, the quantum capacity is given by the optimal rate of using $\mathcal{N}$ to simulate the qubit noiseless channel $\mathrm{id}_{2}$, while the channel simulation cost is given by the optimal rate of using $\mathrm{id}_{2}$ to simulate the channel $\mathcal{N}$. Thus, it operationally holds that

$$
Q_{\mathrm{E}}(\mathcal{N}) \leq Q_{\mathrm{NS}}(\mathcal{N}) \leq S_{\mathrm{NS}}(\mathcal{N}) \leq S_{\mathrm{E}}(\mathcal{N})
$$

where the above four notations represent entanglement-assisted quantum capacity, NS-assisted quantum capacity, NS-assisted quantum simulation cost and entanglement-assisted quantum simulation cost, respectively. The quantum reverse Shannon theorem $[4,5]$ states that the quantum simulation cost is equal to its quantum capacity under entanglement-assistance, i.e., $Q_{\mathrm{E}}(\mathcal{N})=S_{\mathrm{E}}(\mathcal{N})$. In the following, the quantum reverse Shannon theorem under NS-assistance means that $Q_{\mathrm{NS}}(\mathcal{N})=S_{\mathrm{NS}}(\mathcal{N})$.

The AEP of the channel's smooth max-information is the claim that

$$
\lim _{\varepsilon \rightarrow 0} \lim _{n \rightarrow \infty} \frac{1}{n} I_{\max }^{\varepsilon}(A: B)_{\mathcal{N} \otimes n}=I(A: B)_{\mathcal{N}},
$$

where

$$
I(A: B)_{\mathcal{N}}:=\max _{\rho_{A} \in S=(A)} I(A: B)_{\mathcal{N}_{A^{\prime} \rightarrow B}\left(\phi_{A A^{\prime}}\right)}
$$


is the mutual information of the quantum channel and $\phi_{A A^{\prime}}$ is a purification of the state $\rho_{A}$. Based on the operational interpretation in Theorem 6 and the known result that $Q_{\mathrm{E}}(\mathcal{N})=\frac{1}{2} I(A: B)_{\mathcal{N}}[3]$, we have

$$
\operatorname{AEP}(32) \Longleftrightarrow \mathrm{Q}_{\mathrm{E}}(\mathcal{N})=\mathrm{S}_{\mathrm{NS}}(\mathcal{N})
$$

Hence the QRST implies the AEP for the channel's smooth max-information. On the other hand, we can directly prove the AEP in Theorem 8. This proof then implies the QRST in the presence of NS correlations.

In the following, we utilize the smooth max-information of a quantum state and its variation:

$$
\begin{aligned}
& I_{\max }^{\varepsilon}(A: B)_{\rho}:=\inf _{\widetilde{\rho} \approx^{\varepsilon} \rho} I_{\max }(A: B)_{\widetilde{\rho}}, \\
& \widehat{I}_{\max }^{\varepsilon}(A: B)_{\rho}:=\inf _{\substack{\tilde{\rho} \approx^{\varepsilon} \rho \\
\tilde{\rho}_{A}=\rho_{A}}} I_{\max }(A: B)_{\widetilde{\rho}} .
\end{aligned}
$$

The first smooth max-information is most often used in the literature [5, 23]. The second variation naturally appears in our discussion of the channel simulation problem. The restricted smoothing such that the marginal state is fixed comes from the definition of diamond norm where the reference system of the input state is untouched. Using ideas from [36], the following lemma shows that these two quantities are equivalent up to some correction terms. The proof can be found in Appendix B.

Lemma 7 For any quantum state $\rho_{A B}$ and $\varepsilon \in(0,1)$, it holds

$$
\widehat{I}_{\max }^{\varepsilon}(A: B)_{\rho} \leq I_{\max }^{\varepsilon / 6}(A: B)_{\rho}+g(\varepsilon) \text { with } g(\varepsilon)=\log \left(1+72 / \varepsilon^{2}\right) .
$$

Theorem 8 For any quantum channel $\mathcal{N}$ we have the AEP for the channel's smooth max-information:

$$
\lim _{\varepsilon \rightarrow 0} \lim _{n \rightarrow \infty} \frac{1}{n} I_{\max }^{\varepsilon}(A: B)_{\mathcal{N} \otimes n}=I(A: B)_{\mathcal{N}} .
$$

Proof The proof strategy is as follows. We first use the post-selection technique to show that the channel's smooth max-information is upper bounded by the quantity in Eq. (36). By Eq. (37) we can then use the basic properties of the smooth max-information developed in [5] to show one direction of the proof. The other direction can be proved via the continuity of the mutual information of quantum states.

Consider $n$ uses of the channel $\mathcal{N}$ and let $\omega_{R A A^{\prime}}^{n}$ be the purification of the de Finetti state $\omega_{A A^{\prime}}^{n}=$ $\int \sigma_{A A^{\prime}}^{\otimes n} d\left(\sigma_{A A^{\prime}}\right)$ with pure states $\sigma_{A A^{\prime}}=|\sigma\rangle\left\langle\left.\sigma\right|_{A A^{\prime}}\right.$ and $d(\cdot)$ the measure on the normalized pure states induced by the Haar measure. Furthermore we can assume without loss of generality that $|R| \leq$ $(n+1)^{|A|^{2}-1}$. Note that $\omega_{A^{\prime}}^{n}$ is a full rank state. We have the following chain of inequalities

$$
\begin{aligned}
& I_{\max }^{\varepsilon}(A: B)_{\mathcal{N}}^{\otimes n} \\
= & \inf \left\{I_{\max }(R A: B)_{\widetilde{\mathcal{N}}^{n}\left(\omega_{R A A^{\prime}}^{n}\right)} \mid \frac{1}{2}\left\|\tilde{\mathcal{N}}^{n}-\mathcal{N}^{\otimes n}\right\|_{\diamond} \leq \varepsilon, \tilde{\mathcal{N}}^{n} \in \operatorname{CPTP}\left(A^{\prime n}: B^{n}\right)\right\} \\
\leq & \inf \left\{I_{\max }(R A: B)_{\widetilde{\mathcal{N}}^{n}\left(\omega_{R A A^{\prime}}^{n}\right)} \mid \frac{1}{2}\left\|\left(\tilde{\mathcal{N}}^{n}-\mathcal{N}^{\otimes n}\right)\left(\omega_{R A A^{\prime}}^{n}\right)\right\|_{1} \leq \varepsilon_{1}, \tilde{\mathcal{N}}^{n} \in \operatorname{CPTP}\left(A^{\prime n}: B^{n}\right)\right\} \\
\leq & \inf \left\{I_{\max }(R A: B)_{\tilde{\mathcal{N}}^{n}\left(\omega_{R A A^{\prime}}^{n}\right)} \mid \tilde{\mathcal{N}}^{n}\left(\omega_{R A A^{\prime}}^{n}\right) \approx^{\varepsilon_{2}} \mathcal{N}^{\otimes n}\left(\omega_{R A A^{\prime}}^{n}\right), \tilde{\mathcal{N}}^{n} \in \operatorname{CPTP}\left(A^{\prime n}: B^{n}\right)\right\},
\end{aligned}
$$

where $\varepsilon_{1}=\varepsilon(n+1)^{-\left(\left|A^{\prime}\right|^{2}-1\right)}, \varepsilon_{2}=\varepsilon_{1}$. In the first equality, we choose $\omega_{R A A^{\prime}}^{n}$ as the input state of the channel's max-information. In the first inequality, we use post-selection technique (see [5, Prop. D.4]). This relaxes the diamond norm to the trace norm. In the second inequality, we replace the trace norm with the purified distance since $\frac{1}{2}\|\rho-\sigma\|_{1} \leq P(\rho, \sigma)$. 
From Lemma 10 in Appendix B, we know the following two sets are the same,

$$
\begin{aligned}
\left\{\tilde{\mathcal{N}}^{n}\left(\omega_{R A A^{\prime}}^{n}\right) \approx^{\varepsilon_{2}} \mathcal{N}^{\otimes n}\left(\omega_{R A A^{\prime}}^{n}\right) \mid \tilde{\mathcal{N}}^{n}\right. & \left.\in \operatorname{CPTP}\left(A^{\prime n}: B^{n}\right)\right\} \\
& =\left\{\sigma_{R A B}^{n} \approx^{\varepsilon_{2}} \mathcal{N}^{\otimes n}\left(\omega_{R A A^{\prime}}^{n}\right) \mid \sigma_{R A}^{n}=\omega_{R A}^{n}\right\} .
\end{aligned}
$$

Combining Eqs. (39c) and (40), we have

$$
I_{\max }^{\varepsilon}(A: B)_{\mathcal{N} \otimes n} \leq \widehat{I}_{\max }^{\varepsilon_{2}}(R A: B)_{\mathcal{N} \otimes n}\left(\omega_{R A A^{\prime}}^{n}\right) .
$$

From Eq. (37), denote $\varepsilon_{3}=\varepsilon_{2} / 6$, we have

$$
I_{\max }^{\varepsilon}(A: B)_{\mathcal{N} \otimes n} \leq I_{\max }^{\varepsilon_{3}}(R A: B)_{\mathcal{N} \otimes n}\left(\omega_{R A A^{\prime}}^{n}\right)+g\left(\varepsilon_{2}\right) .
$$

Then we can use some known properties of the smooth max-information from [5, 23], which leads to

$$
\begin{aligned}
I_{\max }^{\varepsilon_{3}}(R A: B)_{\mathcal{N} \otimes n}\left(\omega_{R A A^{\prime}}^{n}\right) & \leq I_{\max }^{\varepsilon_{4}}(B: R A)_{\mathcal{N} \otimes n}\left(\omega_{R A A^{\prime}}^{n}\right)+f\left(\varepsilon_{4}, \varepsilon_{4}\right) \\
& \leq I_{\max }^{\varepsilon_{4}}(B: A)_{\mathcal{N} \otimes n}\left(\omega_{A A^{\prime}}^{n}\right)+2 \log |R|+f\left(\varepsilon_{4}, \varepsilon_{4}\right) \\
& =I_{\max }^{\varepsilon_{4}}(B: A)_{\mathcal{N} \otimes n}\left(\sum_{i \in I} p_{i}\left(\sigma_{A A^{\prime}}^{i}\right)^{\otimes n}\right)+2 \log |R|+f\left(\varepsilon_{4}, \varepsilon_{4}\right) \\
& \leq \max _{\sigma_{A A^{\prime}}^{i}} I_{\max }^{\varepsilon_{4}}(B: A)_{\mathcal{N} \otimes n}\left(\left(\sigma_{A A^{\prime}}^{i}\right)^{\otimes n}\right)+\log |I|+2 \log |R|+f\left(\varepsilon_{4}, \varepsilon_{4}\right) \\
& \leq \max _{\sigma_{A A^{\prime}}} I_{\max }^{\varepsilon_{4}}(B: A)_{\mathcal{N} \otimes n}\left(\sigma_{A A^{\prime}}^{\otimes n}\right)+\log |I|+2 \log |R|+f\left(\varepsilon_{4}, \varepsilon_{4}\right)
\end{aligned}
$$

where $\varepsilon_{4}=\varepsilon_{3} / 2, f\left(\varepsilon, \varepsilon^{\prime}\right)=\log \left(\frac{1}{1-\sqrt{1-\varepsilon^{2}}}+\frac{1}{1-\varepsilon^{\prime}}\right)$ and $|I|=(n+1)^{2|A|\left|A^{\prime}\right|-2}$. In the first line, we swap the system order according to [23, Corollary 5]. In the second line, we get rid of purification system $R$ according to [5, Lemma B.12]. In the third line, we express the integral $\omega_{A A^{\prime}}^{n}=\int \sigma_{A A^{\prime}}^{\otimes n} d\left(\sigma_{A A^{\prime}}\right)$ into convex combination of finite number of operators according to [5, Corollary D.6]. In the forth line, we use the quasi-convexity of the smooth max-information [5, Lemma B.21]. In the last line, we relax the maximization to all pure states $\sigma_{A A^{\prime}}$.

Combining Eqs. (42), (43e) and the AEP for the smooth max-information from [5, Lemma B.24], we get

$$
\lim _{\varepsilon \rightarrow 0} \lim _{n \rightarrow \infty} \frac{1}{n} I_{\max }^{\varepsilon}(A: B)_{\mathcal{N} \otimes n} \leq \max _{\sigma_{A A^{\prime}}} I(A: B)_{\mathcal{N}\left(\sigma_{A A^{\prime}}\right)}=I(A: B)_{\mathcal{N}}
$$

On the other hand, for $\rho_{A^{\prime}}$ full rank we find the following chain of inequalities

$$
\begin{aligned}
& I_{\max }^{\varepsilon}(A: B)_{\mathcal{N} \otimes n}=\inf _{\substack{\frac{1}{2}\left\|\widetilde{\mathcal{N}}^{n}-\mathcal{N}^{\otimes n}\right\| \diamond \leq \varepsilon \\
\tilde{\mathcal{N}}^{n} \in \operatorname{CPTP}\left(A^{\prime n}: B^{n}\right)}} \inf _{\sigma_{B}^{n} \in \mathcal{S}_{=}\left(B^{\otimes n}\right)} D_{\max }\left(\widetilde{\mathcal{N}}_{A^{\prime} \rightarrow B}^{n}\left(\phi_{A A^{\prime}}^{\otimes n}\right) \| \phi_{A}^{\otimes n} \otimes \sigma_{B}^{n}\right) \\
& \geq \inf _{\substack{\frac{1}{2}\left\|\tilde{\mathcal{N}}^{n}-\mathcal{N}^{\otimes n}\right\|_{\diamond \leq \varepsilon} \\
\tilde{\mathcal{N}}^{n} \in \operatorname{CPTP}\left(A^{\prime n}: B^{n}\right)}} \inf _{\sigma_{B}^{n} \in \mathcal{S}_{=}\left(B^{\otimes n}\right)} D\left(\tilde{\mathcal{N}}_{A^{\prime} \rightarrow B}^{n}\left(\phi_{A A^{\prime}}^{\otimes n}\right) \| \phi_{A}^{\otimes n} \otimes \sigma_{B}^{n}\right) \\
& =\inf _{\substack{\frac{1}{2}\left\|\tilde{\mathcal{N}}^{n}-\mathcal{N}^{\otimes n}\right\|_{\diamond} \leq \varepsilon \\
\tilde{\mathcal{N}}^{n} \in \operatorname{CPTP}\left(A^{\prime n}: B^{n}\right)}} I(A: B)_{\widetilde{\mathcal{N}}_{A^{\prime} \rightarrow B}^{n}}\left(\phi_{A A^{\prime}}^{\otimes n}\right) \\
& \geq I(A: B)_{\mathcal{N}_{A^{\prime} \rightarrow B}^{\otimes n}\left(\phi_{A A^{\prime}}^{\otimes n}\right)}-\left(8 n \varepsilon \log |A|+2 h_{2}(2 \varepsilon)\right) \\
& =n I(A: B)_{\mathcal{N}_{A^{\prime} \rightarrow B}\left(\phi_{A A^{\prime}}\right)}-\left(8 n \varepsilon \log |A|+2 h_{2}(2 \varepsilon)\right)
\end{aligned}
$$


where $h_{2}(\cdot)$ is the binary entropy. In the second line, we use the fact that max-relative entropy is never smaller than the relative entropy [34]. The third line follows from the definition of the mutual information of a quantum state. The fourth line follows from the continuity of quantum mutual information in Lemma 11 (Appendix B). The fifth line follows from the additivity of quantum mutual information. The last line follows from the assumption that $\phi_{A A^{\prime}}$ is the optimizer of $I(A: B)_{\mathcal{N}}$. By continuity of the quantity the supremum over full rank states $\rho_{A^{\prime}}$ is equal to the quantum mutual information of the channel. Finally, we have

$$
\lim _{\varepsilon \rightarrow 0} \lim _{n \rightarrow \infty} \frac{1}{n} I_{\max }^{\varepsilon}(A: B)_{\mathcal{N} \otimes n} \geq I(A: B)_{\mathcal{N}}
$$

Combining Eqs. (44) and (46), we conclude the claim.

\section{EXAMPLES}

In this section, we apply our results to some basic channels. For classical channels, the one-shot $\varepsilon$-error quantum simulation cost can be given by a linear program as shown in Eq. (58) (Appendix A). Using the symmetry of the quantum depolarizing channel, we can also simplify its $n$-shot simulation cost as a linear program. Moreover, the zero-error simulation cost of various channels can be solved analytically.

Example 1. The quantum depolarizing channel is given by $\mathcal{N}(\rho)=(1-p) \rho+p \cdot \frac{\mathbb{1}}{d}$ with dimension $d$. Its Choi-Jamiołkowski matrix $J_{\mathcal{N}}$ commutes with any local unitary $U \otimes \bar{U}$ and $J_{\mathcal{N}}^{\otimes n}$ is invariant under any permutation of the tensor factors. Exploiting these symmetries, we can simplify the SDP (14) for $\mathcal{N}^{\otimes n}$ to a linear program (61) in Appendix A. Numerical implementation has been shown in Fig. 2. We can see that as the number of channel uses $n$ increases, the average quantum simulation cost will approach to its entanglement-assisted quantum capacity [37], i.e., half of the quantum mutual information of the channel.

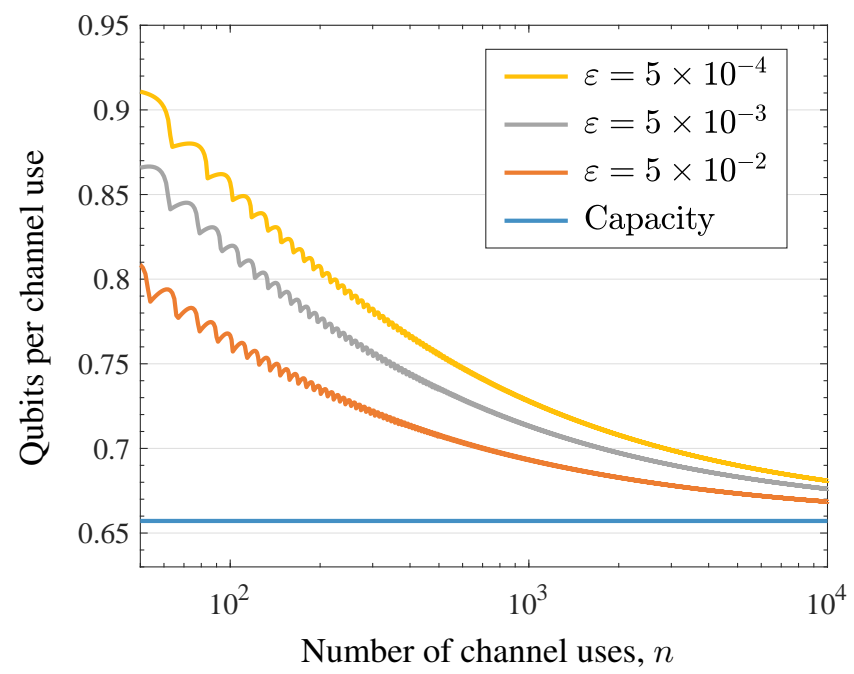

FIG. 2: Exact value by the linear program (61) of the average simulation cost for three different error tolerances $\varepsilon \in\left\{5 \times 10^{-4}, 5 \times 10^{-3}, 5 \times 10^{-2}\right\}$ and the qubit depolarizing channel with failure probability $p=0.15$. The lowest line marks the entanglement-assisted quantum capacity of the channel (roughly 0.657 qubits per channel use). 
Recall that the primal and dual SDPs of the zero-error simulation cost are given by [8]

Primal: $\quad S_{\mathrm{NS}, 0}(\mathcal{N})=S_{\mathrm{NS}, 0}^{(1)}(\mathcal{N})=\frac{1}{2} \log \inf \left\{\operatorname{Tr} V_{B} \mid J_{\mathcal{N}} \leq \mathbb{1}_{A} \otimes V_{B}\right\}$,

Dual: $\quad S_{\mathrm{NS}, 0}(\mathcal{N})=S_{\mathrm{NS}, 0}^{(1)}(\mathcal{N})=\frac{1}{2} \log \sup \left\{\operatorname{Tr} J_{\mathcal{N}} X_{A B} \mid \operatorname{Tr}_{A} X_{A B} \leq \mathbb{1}_{B}, X_{A B} \geq 0\right\}$.

We study some fundamental channels and show their analytical solutions by explicitly constructing feasible solutions in both primal and dual problems, respectively. Using the weak duality, we can argue that the feasible solutions we construct are optimal.

Example 2. The quantum depolarizing channel is $\mathcal{N}(\rho)=(1-p) \rho+p \cdot \frac{\mathbb{1}}{d}$ with dimension $d$. Taking

$$
V_{B}=\left(d(1-p)+\frac{p}{d}\right) \mathbb{1}_{B}, \quad \text { and } \quad X_{A B}=\sum_{i, j=0}^{d-1}|i i\rangle\langle j j|,
$$

in the primal and dual problems respectively, we can verify that they are feasible solutions. Thus, we have

$$
\frac{1}{2} \log \left(d^{2}(1-p)+p\right)=\frac{1}{2} \log \operatorname{Tr} J_{\mathcal{N}} X_{A B} \leq S_{\mathrm{NS}, 0}(\mathcal{N}) \leq \frac{1}{2} \log \operatorname{Tr} V_{B}=\frac{1}{2} \log \left(d^{2}(1-p)+p\right) .
$$

We find that

$$
S_{\mathrm{NS}, 0}(\mathcal{N})=\frac{1}{2} \log \left(d^{2}(1-p)+p\right)
$$

Example 3. The amplitude damping channel is $\mathcal{N}(\rho)=\sum_{i=0}^{1} E_{i} \rho E_{i}^{\dagger}$ with $E_{0}=|0\rangle\langle 0|+\sqrt{1-r}| 1\rangle\langle 1|$, $E_{1}=\sqrt{r}|0\rangle\langle 1|$ and $0 \leq r \leq 1$. The optimal solutions are given by

$$
V_{B}=(1+\sqrt{1-r})|0\rangle\langle 0|+(\sqrt{1-r}+1-r)| 1\rangle\langle 1| \text { and } X_{A B}=(|00\rangle+|11\rangle)(\langle 00|+\langle 11|) .
$$

We find that

$$
S_{\mathrm{NS}, 0}(\mathcal{N})=\frac{1}{2} \log (2(1+\sqrt{1-r})-r)
$$

Example 4. The dephasing channel is $\mathcal{N}(\rho)=(1-p) \rho+p Z \rho Z$ with $Z=|0\rangle\langle 0|-| 1\rangle\langle 1|$. The optimal solutions are given by

$$
V_{B}=(|2 p-1|+1) \mathbb{1}_{B} \quad \text { and } \quad X_{A B}=(|00\rangle+|11\rangle)(\langle 00|+\langle 11|) .
$$

We find that

$$
S_{\mathrm{NS}, 0}(\mathcal{N})=\frac{1}{2} \log (|4 p-2|+2)
$$

Example 5. The quantum erasure channel is $\mathcal{N}(\rho)=(1-p) \rho+p|e\rangle\langle e|$ with $|e\rangle$ orthogonal to the input Hilbert space. The optimal solutions are given by

$$
V_{B}=d(1-p) \sum_{i, j=0}^{d-1}|i\rangle\langle i|+p| d\rangle\langle d| \quad \text { and } \quad X_{A B}=\sum_{i, j=0}^{d-1}|i i\rangle\left\langle j j\left|+\frac{1}{d} \sum_{i=0}^{d-1}\right| i\right\rangle\langle i|\otimes| d\rangle\langle d| .
$$

We find that

$$
S_{\mathrm{NS}, 0}(\mathcal{N})=\frac{1}{2} \log \left(d^{2}(1-p)+p\right)
$$




\section{DISCUSSION}

Since the entanglement-assisted capacity allows a single-letter characterization, it is natural to consider a second-order refinement thereof. A second-order expansion of an achievable rate was established in [38] but no matching second-order converse bound is known. Our one-shot NS-assisted quantum simulation cost and the channel's smooth max-information may provide some insights in this direction.

Note that the channel simulation cost operationally provides converse for the channel capacity. However this approach does not provide a tighter bound than the NS-assisted capacity in the one-shot and asymptotic setting (see, e.g., $[18,26,28]$ ).

\section{Acknowledgements}

We are grateful to Runyao Duan for discussions. MB thanks the Centre for Quantum Software and Information at the University of Technology Sydney for their hospitality while part of this work was done. MT acknowledges an Australian Research Council Discovery Early Career Researcher Award, project No. DE160100821.

[1] D. Kretschmann and R. F. Werner, "Tema con variazioni: Quantum channel capacity," New Journal of Physics, vol. 6, 2004.

[2] C. E. Shannon, “A mathematical theory of communication," The Bell System Technical Journal, vol. 27, no. 3, pp. 379-423, 1948.

[3] C. H. Bennett, P. W. Shor, J. A. Smolin, and A. V. Thapliyal, "Entanglement-assisted capacity of a quantum channel and the reverse Shannon theorem," IEEE Transactions on Information Theory, vol. 48, no. 10, pp. 2637-2655, 2002.

[4] C. H. Bennett, I. Devetak, A. W. Harrow, P. W. Shor, and A. Winter, "The Quantum Reverse Shannon Theorem and Resource Tradeoffs for Simulating Quantum Channels," IEEE Transactions on Information Theory, vol. 60, no. 5, pp. 2926-2959, 2014.

[5] M. Berta, M. Christandl, and R. Renner, "The quantum reverse Shannon theorem based on one-shot information theory," Communications in Mathematical Physics, vol. 306, no. 3, pp. 579-615, 2011.

[6] C. E. Shannon, "The zero error capacity of a noisy channel," IRE Transactions on Information Theory, vol. 2, no. 3, pp. 8-19, 1956.

[7] T. S. Cubitt, D. Leung, W. Matthews, and A. Winter, "Zero-Error Channel Capacity and Simulation Assisted by Non-Local Correlations," IEEE Transactions on Information Theory, vol. 57, no. 8, pp. 5509-5523, 2011.

[8] R. Duan and A. Winter, "No-Signalling-Assisted Zero-Error Capacity of Quantum Channels and an Information Theoretic Interpretation of the Lovász Number," IEEE Transactions on Information Theory, vol. 62, no. 2, pp. 891-914, 2016.

[9] X. Wang and R. Duan, "On the quantum no-signalling assisted zero-error classical simulation cost of noncommutative bipartite graphs," in IEEE ISIT, 2016, pp. 2244-2248.

[10] M. Berta, F. G. S. L. Brandao, M. Christandl, and S. Wehner, "Entanglement Cost of Quantum Channels," IEEE Transactions on Information Theory, vol. 59, no. 10, pp. 6779-6795, 2013.

[11] K. Ben Dana, M. García Díaz, M. Mejatty, and A. Winter, "Resource theory of coherence: Beyond states," Physical Review A, vol. 95, no. 6, p. 062327, 2017.

[12] Y. Polyanskiy, H. V. Poor, and S. Verdú, "Channel coding rate in the finite blocklength regime," IEEE Transactions on Information Theory, vol. 56, no. 5, pp. 2307-2359, 2010.

[13] M. Hayashi, "Information spectrum approach to second-order coding rate in channel coding," IEEE Transactions on Information Theory, vol. 55, no. 11, pp. 4947-4966, 2009.

[14] M. Tomamichel, "A framework for non-asymptotic quantum information theory," arXiv:1203.2142, 2012.

[15] N. Datta and M.-H. Hsieh, "One-shot entanglement-assisted quantum and classical communication," IEEE Transactions on Information Theory, vol. 59, no. 3, pp. 1929-1939, 2013. 
[16] F. Buscemi and N. Datta, "Distilling entanglement from arbitrary resources," Journal of Mathematical Physics, vol. 51, no. 10, p. 102201, 2010.

[17] W. Matthews and S. Wehner, "Finite blocklength converse bounds for quantum channels," IEEE Transactions on Information Theory, vol. 60, no. 11, pp. 7317-7329, 2014.

[18] X. Wang, W. Xie, and R. Duan, "Semidefinite programming strong converse bounds for classical capacity," IEEE Transactions on Information Theory, vol. 64, no. 1, pp. 640-653, 2018.

[19] J. M. Renes and R. Renner, "Noisy channel coding via privacy amplification and information reconciliation," IEEE Transactions on Information Theory, vol. 57, no. 11, pp. 7377-7385, 2011.

[20] N. Datta, J. M. Renes, R. Renner, and M. M. Wilde, "One-Shot Lossy Quantum Data Compression," IEEE Transactions on Information Theory, vol. 59, no. 12, pp. 8057-8076, 2013.

[21] M. Berta, M. Christandl, and D. Touchette, "Smooth Entropy Bounds on One-Shot Quantum State Redistribution," IEEE Transactions on Information Theory, vol. 62, no. 3, pp. 1425-1439, 2016.

[22] A. Anshu, R. Jain, P. Mukhopadhyay, A. Shayeghi, and P. Yao, "New One Shot Quantum Protocols With Application to Communication Complexity," IEEE Transactions on Information Theory, vol. 62, no. 12, pp. 7566$7577,2016$.

[23] N. Ciganovic, N. J. Beaudry, and R. Renner, "Smooth Max-Information as One-Shot Generalization for Mutual Information," IEEE Transactions on Information Theory, vol. 60, no. 3, pp. 1573-1581, 2014.

[24] L. Vandenberghe and S. Boyd, "Semidefinite Programming," SIAM Review, vol. 38, no. 1, pp. 49-95, 1996.

[25] X. Wang, K. Fang, and M. Tomamichel, "On converse bounds for classical communication over quantum channels," arXiv:1709.05258, 2017.

[26] W. Matthews, "A Linear Program for the Finite Block Length Converse of Polyanskiy-Poor-Verdú Via Nonsignaling Codes," IEEE Transactions on Information Theory, vol. 58, no. 12, pp. 7036-7044, 2012.

[27] G. Chiribella, G. M. D'Ariano, and P. Perinotti, "Transforming quantum operations: Quantum supermaps," $E P L$ (Europhysics Letters), vol. 83, no. 3, p. 30004, 2008.

[28] D. Leung and W. Matthews, "On the Power of PPT-Preserving and Non-Signalling Codes," IEEE Transactions on Information Theory, vol. 61, no. 8, pp. 4486-4499, 2015.

[29] K. Audenaert, M. B. Plenio, and J. Eisert, "Entanglement Cost under Positive-Partial-Transpose-Preserving Operations," Physical Review Letters, vol. 90, no. 2, p. 027901, 2003.

[30] X. Wang, K. Fang, and R. Duan, "Semidefinite programming converse bounds for quantum communication," arXiv:1709.00200, 2017.

[31] E. M. Rains, “A semidefinite program for distillable entanglement," IEEE Transactions on Information Theory, vol. 47, no. 7, pp. 2921-2933, 2001.

[32] K. Fang, X. Wang, M. Tomamichel, and R. Duan, "Non-asymptotic entanglement distillation," arXiv:1706.06221, 2017.

[33] J. Watrous, "Semidefinite Programs for Completely Bounded Norms," Theory of Computing, vol. 5, no. 1, pp. 217-238, 2009.

[34] N. Datta, "Min-and max-relative entropies and a new entanglement monotone," IEEE Transactions on Information Theory, vol. 55, no. 6, pp. 2816-2826, 2009.

[35] R. Renner, "Security of quantum key distribution," PhD thesis, ETH Zurich, vol. 6, no. 01, pp. 1-127, 2005.

[36] A. Anshu, M. Berta, R. Jain, and M. Tomamichel, To appear, 2018.

[37] C. H. Bennett, P. W. Shor, J. A. Smolin, and A. V. Thapliyal, "Entanglement-assisted classical capacity of noisy quantum channels," Physical Review Letters, vol. 83, no. 15, p. 3081, 1999.

[38] N. Datta, M. Tomamichel, and M. M. Wilde, "On the second-order asymptotics for entanglement-assisted communication," Quantum Information Processing, pp. 1-23, 2016.

[39] A. Uhlmann, "The "transition probability" in the state space of a *-algebra," Reports on Mathematical Physics, vol. 9, no. 2, pp. 273-279, 1976.

[40] R. Alicki and M. Fannes, "Continuity of quantum conditional information," Journal of Physics A: Mathematical and General, vol. 37, no. 5, pp. L55-L57, feb 2004. 


\section{Appendix A LINEAR PROGRAMS}

For any classical channel $\mathcal{N}(y \mid x)$, its one-shot simulation cost is given by a linear program,

$$
\begin{aligned}
S_{\mathrm{NS}, \varepsilon}^{(1)}(\mathcal{N})=\frac{1}{2} \log \inf & \sum V_{y} \\
\text { s.t. } \quad & Y_{x y} \geq \widetilde{\mathcal{N}}(y \mid x)-\mathcal{N}(y \mid x), Y_{x y} \geq 0, \forall x, y, \\
& \widetilde{\mathcal{N}}(y \mid x) \geq 0, \forall x, y, \sum_{y} \widetilde{\mathcal{N}}(y \mid x)=1, \forall x, \\
& \widetilde{\mathcal{N}}(y \mid x) \leq V_{y}, \forall x, y, \sum_{y} Y_{x y} \leq \varepsilon, \forall x .
\end{aligned}
$$

For the quantum depolarizing channel $\mathcal{N}(\rho)=(1-p) \rho+p \cdot \frac{\mathbb{1}}{d}$, its Choi-Jamiołkowski matrix is given by $J_{\mathcal{N}}=q_{1} \Phi_{d}+q_{2} \Phi_{d}^{\perp}$ where $q_{1}=d(1-p)+\frac{p}{d}, q_{2}=\frac{p}{d}$ and $\Phi_{d}$ is the maximally entangled state with dimension $d, \Phi_{d}^{\perp}=\mathbb{1}-\Phi_{d}$. Then we have

$$
J_{\mathcal{N}}^{\otimes n}=\sum_{k=0}^{n} p_{k} P_{k}^{n}\left(\Phi_{d}, \Phi_{d}^{\perp}\right) \quad \text { with } \quad p_{k}=q_{1}^{k} q_{2}^{n-k},
$$

and $P_{k}^{n}\left(\Phi_{d}, \Phi_{d}^{\perp}\right)$ denotes the summation of $n$-fold tensor products of $\Phi_{d}$ and $\Phi_{d}^{\perp}$ with exactly $k$-fold of $\Phi_{d}$. For example, $P_{1}^{3}\left(\Phi_{d}, \Phi_{d}^{\perp}\right)=\Phi_{d}^{\perp} \otimes \Phi_{d}^{\perp} \otimes \Phi_{d}+\Phi_{d}^{\perp} \otimes \Phi_{d} \otimes \Phi_{d}^{\perp}+\Phi_{d} \otimes \Phi_{d}^{\perp} \otimes \Phi_{d}^{\perp}$. Due to the symmetries of $J_{\mathcal{N}}^{\otimes n}$, we can take the optimal solution in SDP (14) in form of

$$
J_{\widetilde{\mathcal{N}}^{n}}=\sum_{k=0}^{n} r_{k} P_{k}^{n}\left(\Phi_{d}, \Phi_{d}^{\perp}\right), \quad Y=\sum_{k=0}^{n} y_{k} P_{k}^{n}\left(\Phi_{d}, \Phi_{d}^{\perp}\right), \quad \text { and } \quad V=s \mathbb{1} .
$$

Then we have the LP as follows,

$$
\begin{aligned}
S_{\mathrm{NS}, \varepsilon}^{(1)}\left(\mathcal{N}^{\otimes n}\right)=\frac{1}{2} \log \inf \quad & d^{n} \cdot s \\
\text { s.t. } \quad & y_{k}-r_{k}+p_{k} \geq 0, y_{k} \geq 0,0 \leq r_{k} \leq s, \forall k \\
& \sum_{k=0}^{n}\left(\begin{array}{l}
n \\
k
\end{array}\right)\left(\frac{1}{d}\right)^{k}\left(d-\frac{1}{d}\right)^{n-k} r_{k}=1, \\
& \sum_{k=0}^{n}\left(\begin{array}{l}
n \\
k
\end{array}\right)\left(\frac{1}{d}\right)^{k}\left(d-\frac{1}{d}\right)^{n-k} y_{k} \leq \varepsilon .
\end{aligned}
$$

\section{Appendix B TECHNICAL LEMMAS}

Lemma 9 For any quantum state $\rho_{A B}$ and $\varepsilon \in(0,1)$, it holds

$$
\widehat{I}_{\max }^{\varepsilon}(A: B)_{\rho} \leq I_{\max }^{\varepsilon / 6}(A: B)_{\rho}+g(\varepsilon) \text { with } g(\varepsilon)=\log \left(1+72 / \varepsilon^{2}\right) .
$$

Proof Recall the definitions of the smooth max-information for quantum states:

$$
\begin{gathered}
\widehat{I}_{\max }^{\varepsilon}(A: B)_{\rho}=\inf _{\substack{\tilde{\rho} \approx^{\varepsilon} \rho \\
\tilde{\rho}_{A}=\rho_{A}}} \inf _{\sigma_{B}} D_{\max }\left(\widetilde{\rho}_{A B} \| \rho_{A} \otimes \sigma_{B}\right), \\
I_{\max }^{\varepsilon}(A: B)_{\rho}=\inf _{\widetilde{\rho} \approx^{\varepsilon} \rho \sigma_{B}} \inf _{\max }\left(\widetilde{\rho}_{A B} \| \widetilde{\rho}_{A} \otimes \sigma_{B}\right) .
\end{gathered}
$$


In [36] the authors also discuss the variation

$$
\widetilde{I}_{\max }^{\varepsilon}(A: B)_{\rho}=\inf _{\widetilde{\rho} \approx^{\varepsilon} \rho \sigma_{B}} D_{\max }\left(\widetilde{\rho}_{A B} \| \rho_{A} \otimes \sigma_{B}\right),
$$

where the marginal state in the second term is fixed to be $\rho_{A}$. It was shown in [36] that for any quantum state $\rho_{A B}$ and $\varepsilon \in(0,1)$ we have

$$
\widehat{I}_{\max }^{\varepsilon}(A: B)_{\rho} \leq \widetilde{I}_{\max }^{\varepsilon / 3}(A: B)_{\rho}+\log \left(1+72 / \varepsilon^{2}\right) .
$$

To show the result as Eq. (62), we only need to prove

$$
\widetilde{I}_{\max }^{\varepsilon}(A: B)_{\rho} \leq I_{\max }^{\varepsilon / 2}(A: B)_{\rho} .
$$

Denote $I_{\max }^{\varepsilon / 2}(A: B)_{\rho}=\lambda$ and suppose the optimal solution is taken at $\bar{\rho}_{A B}$ and $\sigma_{B}$. Let

$$
\widetilde{\rho}_{A B}=\rho_{A}^{\frac{1}{2}} V_{A} \bar{\rho}_{A}^{-\frac{1}{2}} \bar{\rho}_{A B} \bar{\rho}_{A}^{-\frac{1}{2}} V_{A}^{\dagger} \rho_{A}^{\frac{1}{2}},
$$

where $V_{A}$ is the unitary such that $F\left(\rho_{A}, \bar{\rho}_{A}\right)=\operatorname{Tr} \rho_{A}^{\frac{1}{2}} \bar{\rho}_{A}^{\frac{1}{2}} V_{A}$. By direct calculation, we have

$$
\widetilde{\rho}_{A B}=\rho_{A}^{\frac{1}{2}} V_{A} \bar{\rho}_{A}^{-\frac{1}{2}} \bar{\rho}_{A B} \bar{\rho}_{A}^{-\frac{1}{2}} V_{A}^{\dagger} \rho_{A}^{\frac{1}{2}} \leq 2^{\lambda} \cdot \rho_{A}^{\frac{1}{2}} V_{A} P_{\bar{\rho}_{A}} V_{A}^{\dagger} \rho_{A}^{\frac{1}{2}} \otimes \sigma_{B} \leq 2^{\lambda} \rho_{A} \otimes \sigma_{B},
$$

where $P_{\bar{\rho}_{A}}$ is the projector on the support of $\bar{\rho}_{A}$. Then Eq. (67) follows as soon as we show $\widetilde{\rho}_{A B} \approx^{\varepsilon} \rho_{A B}$. Following the same steps in [36], we know that $P\left(\widetilde{\rho}_{A B}, \bar{\rho}_{A B}\right) \leq P\left(\rho_{A}, \bar{\rho}_{A}\right)$. Thus it holds

$$
\begin{aligned}
P\left(\widetilde{\rho}_{A B}, \rho_{A B}\right) & \leq P\left(\widetilde{\rho}_{A B}, \bar{\rho}_{A B}\right)+P\left(\bar{\rho}_{A B}, \rho_{A B}\right) \\
& \leq P\left(\rho_{A}, \bar{\rho}_{A}\right)+P\left(\bar{\rho}_{A B}, \rho_{A B}\right) \leq 2 P\left(\bar{\rho}_{A B}, \rho_{A B}\right) \leq \varepsilon,
\end{aligned}
$$

which completes the proof.

Lemma 10 For any pure state $\phi_{A A^{\prime}}$ and quantum state $\rho_{A B}$ such that $\phi_{A}=\rho_{A}$, the following two sets are the same,

$$
\left\{\mathcal{N}_{A^{\prime} \rightarrow B}\left(\phi_{A A^{\prime}}\right) \approx^{\varepsilon} \rho_{A B} \mid \mathcal{N} \in \operatorname{CPTP}\left(\mathrm{A}^{\prime}: \mathrm{B}\right)\right\}=\left\{\sigma_{A B} \approx^{\varepsilon} \rho_{A B} \mid \sigma_{A}=\rho_{A}\right\} .
$$

Proof Denote the L.H.S and R.H.S as $\mathcal{S}_{1}$ and $\mathcal{S}_{2}$ respectively. It is clear that $\mathcal{S}_{1} \subseteq \mathcal{S}_{2}$ and we now show the other direction. For any quantum state $\sigma_{A B} \in \mathcal{S}_{2}$, denote $\bar{\sigma}_{A B}=\sigma_{A}^{-1 / 2} \sigma_{A B} \sigma_{A}^{-1 / 2}$. Then, we have $\bar{\sigma}_{A B} \geq 0$ and $\bar{\sigma}_{A}=\mathbb{1}_{A}$. From the Choi-Jamiołkowski isomorphism, we know that there exists a CPTP map $\mathcal{N}_{A^{\prime} \rightarrow B}$ such that $\bar{\sigma}_{A B}=\mathcal{N}_{A^{\prime} \rightarrow B}\left(\Phi_{A A^{\prime}}\right)$, where $\Phi_{A A^{\prime}}$ denotes the un-normalized maximally entangled state. Thus, we get $\sigma_{A B}=\mathcal{N}_{A^{\prime} \rightarrow B}\left(\sigma_{A}^{1 / 2} \Phi_{A A^{\prime}} \sigma_{A}^{1 / 2}\right)$. Denoting $\psi_{A A^{\prime}}=\sigma_{A}^{1 / 2} \Phi_{A A^{\prime}} \sigma_{A}^{1 / 2}$, we have that $\psi_{A A^{\prime}}$ is a purification of $\sigma_{A}$ and since $\sigma_{A}=\rho_{A}=\phi_{A}$ we get that $\phi_{A A^{\prime}}$ is also a purification of $\sigma_{A}$. Due to Uhlmann's theorem [39], there exists a unitary $U$ on the system $A^{\prime}$ such that $\psi_{A A^{\prime}}=\mathcal{U}\left(\phi_{A A^{\prime}}\right)$ with $\mathcal{U}(\cdot)=U \cdot U^{\dagger}$. Hence, we find $\sigma_{A B}=\mathcal{N} \circ \mathcal{U}\left(\phi_{A A^{\prime}}\right) \in \mathcal{S}_{1}$. This completes the proof.

Lemma 11 For any quantum states $\rho_{A B}$ and $\sigma_{A B}$ such that $\rho_{A}=\sigma_{A}$ and $\frac{1}{2}\|\rho-\sigma\|_{1} \leq \varepsilon$, it holds that

$$
\left|I(A: B)_{\rho}-I(A: B)_{\sigma}\right| \leq 8 \varepsilon \log |A|+2 h_{2}(2 \varepsilon),
$$

where $h_{2}(\cdot)$ is the binary entropy.

Proof Since $I(A: B)_{\rho}=H(A)_{\rho}-H(A \mid B)_{\rho}$, we have

$$
\left|I(A: B)_{\rho}-I(A: B)_{\sigma}\right|=\left|H(A \mid B)_{\rho}-H(A \mid B)_{\sigma}\right| \leq 8 \varepsilon \log |A|+2 h_{2}(2 \varepsilon),
$$

where $H(A)$ and $H(A \mid B)$ are von Neumann entropy and conditional entropy respectively. The second inequality follows from the Alicki-Fannes inequality [40]. 